\title{
Perfil das intoxicações exógenas infantis atendidas em um hospital especializado da rede pública de Goiânia-GO'
}

\author{
Perfil de las intoxicaciones infantiles atendidas en un hospital \\ especializado de la red publica de Goiânia-GO
}

\author{
Profile of the infantile exogenous intoxications assisted in a \\ specialized hospital of Goiânia-Go
}

\begin{abstract}
Karina Machado Siqueira', Janaína Ramalho Brandão", Helena Fontenele Limal"I, Ana Claudia de Andrade Garcialv, Flávia Marques Gratonev, Marislei de Sousa Espíndula Brasileiro ${ }^{\mathrm{VI}}$
\end{abstract}

\begin{abstract}
RESUMO
Este estudo objetivou descrever o perfil das intoxicações exógenas agudas, entre crianças de 0 a 12 anos de idade, atendidas em um hospital especializado em atendimentos maternos e infantis da rede pública de Goiânia, entre julho e dezembro de 2006. Trata-se de estudo do tipo descritivo, exploratório, com abordagem quantitativa. A análise demonstrou que a maioria das crianças vive em zona urbana, sendo 53,7\% procedentes de GoiâniaGO. Dentre os 121 casos, $51,2 \%$ corresponderam a intoxicações com indivíduos do sexo masculino, sendo que em $80,2 \%$ o acidente ocorreu no domicílio. A faixa etária mais freqüente correspondeu a crianças entre 1 e 3 anos, totalizando $64,4 \%$ dos casos notificados. A via de intoxicação mais freqüente foi a oral, sendo exclusiva em 116 casos $(95,7 \%)$. O agente causal mais prevalente foi o medicamento (34\%), seguido dos raticidas (14\%) e produtos químicos de uso domiciliar $(13 \%)$. Entende-se que conhecendo o perfil das intoxicações infantis é possível constituir subsídios que permitam trabalhar aspectos preventivos e terapêuticos destas intoxicações. Neste contexto, o enfermeiro assume um papel fundamental, atuando como educador junto aos pais e crianças, com o intuito de reduzir índices de mortalidade e minimizando aspectos relativos à morbidade deste tipo de acidente infantil.
\end{abstract}

Palavras chave: Enfermagem pediátrica; Envenenamento; Emergências.

\section{ABSTRACT}

This study aimed to describe the profile of the acute exogenous intoxications, among children from 0 to 12 years of age, assisted in a specialized hospital of Goiânia between July and December of 2006. It is a study of the type descriptive, exploratory, with quantitative approach. The analysis demonstrated that most of the children lives in urban zone, being 53,7\% coming from Goiânia-GO. Among the 121 cases, $51,2 \%$ corresponded to intoxications with individuals of the masculine sex, and in $80,2 \%$ the accident happened at home. The most frequent age group corresponded to children between 1 and 3 years, totaling $64,4 \%$ of the notified cases. The most common way of intoxication was oral, being exclusive in 116 cases $(95,7 \%)$. The most common causal agent was the medicine (34\%), followed by the mouse poisons $(14 \%)$ and chemical products of household use $(13 \%)$. Understands that knowing the profile of the infantile intoxications is possible to constitute subsidies that allow to work preventive and therapeutic aspects of these intoxications. In this context, the nurse takes a fundamental function, acting like as educator near parents and children, with the intention of reducing mortality rate and

\footnotetext{
Trabalho de Conclusão do Curso de Especialização em Enfermagem Neonatológica e Pediátrica do Centro de Estudos de Enfermagem e Nutrição da Universidade Católica de Goiás.

Enfermeira. Mestre em Enfermagem. Especialista em Enfermagem Neonatológica e Pediátrica. Professora Assistente da Faculdade de Enfermagem da Universidade Federal de Goiás. E-mail: karinams@fen.ufg.br

"Enfermeira. Especialista em Enfermagem Neonatológica e Pediátrica. Supervisora do Pronto-Socorro de Pediatria do Hospital Materno Infantil de Goiânia-GO.

III Enfermeira. Especialista em Enfermagem Neonatológica e Pediátrica. Supervisora do Hospital São Salvador de Goiânia-GO.

IV Enfermeira. Especialista em Enfermagem Neonatológica e Pediátrica. Professora da Faculdade Latino-americana. Supervisora da UTI Neonatal da Santa Casa de Misericórdia de Anápolis-GO.

${ }^{\vee}$ Enfermeira. Especialista em Enfermagem Neonatológica e Pediátrica. Enfermeira do Programa Saúde da Família do município de São Luis dos Montes Belos-GO.

vi Enfermeira. Mestre em Enfermagem. Professora do Centro de Estudos de Enfermagem e Nutrição da Universidade Católica de Goiás. Coordenadora do Curso de Graduação em Enfermagem da Universidade Paulista.
} 
Siqueira KM, Brandão JR, Lima HF, Garcia ACA, Gratone FM, Brasileiro MSE. Perfil das intoxicações exógenas infantis atendidas em um hospital especializado da rede pública de Goiânia-GO. Rev. Eletr. Enf. [Internet]. 2008;10(3):662-72. Available from: http://www.fen.ufg.br/revista/v10/n3/v10n3a12.htm

minimizing relative aspects to the morbid of this type of infantile accident.

Key words: Pediatric nursing; Poisoning; Emergencies.

\section{RESUMEN}

Este estudio tuvo como objetivo describir lo perfil de las intoxicaciones exógenas agudas, entre los niños de 0 a 12 años de edad, atendidos en un hospital especializado de la red publica de Goiânia entre julio y diciembre de 2006. Es un estudio del tipo descriptivo, exploratorio, con abordaje cuantitativo. El análisis demostró que la mayoría de los niños viven en la zona urbana, siendo $53,7 \%$ procedentes de Goiânia-GO. Entre los 121 casos, $51,2 \%$ correspondieron a intoxicaciones con individuos del sexo masculino, y en $80,2 \%$ el accidente ocurrió en el domicilio. El grupo de

\section{NTRODUÇÃO}

O Brasil experimentou, nas últimas décadas, importantes avanços em sua situação de saúde, tais como, a queda da taxa de mortalidade infantil, a redução na mortalidade por doenças infecciosas e o aumento da expectativa de vida. No entanto, algumas questões continuam sendo objetos de preocupação para os profissionais de saúde. Dentre estas, destaca-se o crescimento das causas externas enquanto fator de morbimortalidade, o qual passou a ocupar o segundo lugar entre as causas de morte no país desde 1980. No Brasil, no ano de 2004, ocorreram 127.470 mortes por causas externas, com representatividade importante no total de óbitos ${ }^{(1-2)}$.

Embora a Classificação Internacional de Doenças 10a Revisão (CID-10) use a denominação acidente para alguns tipos de causas externas, a literatura internacional mais recente tem evitado essa denominação, uma vez que essa palavra é comumente associada a um evento não previsível, não passível de prevenção. Dentre as causas externas descritas na CID-10, encontram-se as seguintes nomenclaturas: intoxicações por drogas, medicamentos e substâncias biológicas; efeitos tóxicos de substâncias de origem predominantemente não medicinal; e seqüelas edad más frecuente correspondió a los niños entre 1 y 3 años, totalizando 64,4\% de los casos notificados. La vía más común de intoxicación fue oral, siendo exclusivo en 116 casos $(95,7 \%)$. El agente causal más común fue la medicina (34\%), seguido por venenos del ratón (14\%) y productos químicos de uso domiciliar (13\%). Entiende que sabiendo el perfil de las intoxicaciones infantiles es posible constituir subsidios que permiten trabajar aspectos preventivos y terapéuticos de estas intoxicaciones. En este contexto, el enfermero asume un papel fundamental, actuando como educador cerca de los padres y niños, con la intención de reducir los índices de mortalidad y minimizando los aspectos relativos al mórbido de este tipo de accidente infantil.

Palabras clave: Enfermería pediátrica; Envenenamiento; Urgencias médicas.

de traumatismos, intoxicações e outras conseqüências das causas externas ${ }^{(2)}$.

No Brasil, a intoxicação aguda constitui importante problema de saúde pública, particularmente na faixa etária pediátrica. Medicamentos são os principais agentes responsáveis, seguidos muito de perto pelas intoxicações por animais peçonhentos. De acordo com os dados da rede do Sistema Nacional de Informações Tóxico-Farmacológicas - SINITOX, da Fundação Oswaldo Cruz (FIOCRUZ), no ano de 2000 ocorreram 8.904 notificações não intencionais por medicamentos $^{(3)}$.

Seguem-se, de modo expressivo, as intoxicações por produtos domissanitários, pesticidas e produtos químicos de uso industrial $^{(3)}$. Em detrimento ao desenvolvimento de campanhas sobre prevenção de acidentes na infância, o número de crianças que procuram os serviços de saúde para tratamento de intoxicações exógenas agudas é bastante elevado, o que gera preocupações junto aos profissionais de saúde.

$O$ interesse em se pesquisar sobre o perfil das intoxicações infantis surgiu ao se observar a significativa demanda de atendimento a crianças com idade entre 0 e 12 anos no serviço de pronto atendimento do Hospital Materno Infantil de Goiânia, vítimas de causas externas caracterizadas como intoxicações. Acredita-se 
Siqueira KM, Brandão JR, Lima HF, Garcia ACA, Gratone FM, Brasileiro MSE. Perfil das intoxicações exógenas infantis atendidas em um hospital especializado da rede pública de Goiânia-GO. Rev. Eletr. Enf. [Internet]. 2008;10(3):662-72. Available from: http://www.fen.ufg.br/revista/v10/n3/v10n3a12.htm

que o desenvolvimento de pesquisas que caracterizem o perfil desses acidentes possa subsidiar estratégias de prevenção que visem reduzir os índices de morbimortalidade infantil decorrentes do contato com substâncias tóxicas.

A Convenção sobre os Direitos da Criança e do Adolescente do Brasil normatiza que devese assegurar a todos os setores da sociedade, em especial aos pais e as crianças, o conhecimento dos princípios básicos de saúde e, entre outros, o de ações voltadas para a prevenção de acidentes, recebendo apoio para a aplicação destes conhecimentos. Fica, portanto, evidente a necessidade de investimentos na prevenção de acidentes, e o primeiro passo neste sentido seria modificar o conceito de que os acidentes estão relacionados com casualidade e imprevisibilidade ${ }^{(4)}$.

Intoxicações exógenas agudas podem ser definidas como as conseqüências clínicas e/ou bioquímicas da exposição aguda a substâncias químicas encontradas no ambiente, como ar, água, alimentos, plantas, animais, ou isoladas, como pesticidas, medicamentos, produtos de uso industrial e uso domiciliar, entre outros ${ }^{(5)}$.

A maioria das intoxicações ocorridas na infância está vinculada a situações consideradas facilitadoras, incluindo as características peculiares à faixa etária infantil, os comportamentos inadequados da família ou outras pessoas próximas e o pouco incentivo às estratégias de prevenção. Em muitos casos, o que se observa é que as estratégias de prevenção são pouco valorizadas e o acidente é visto como algo comum e inevitável à infância.

As principais causas para o contato de crianças com produtos considerados tóxicos são o armazenamento incorreto e a supervisão inadequada. As intoxicações em crianças são mais freqüentes em casas desorganizadas ou mal arrumadas e em famílias numerosas, com número superior a três crianças ${ }^{(5-6)}$. Entretanto, os acidentes por intoxicação nem sempre podem se constituir negligência do adulto responsável, pois as crianças, após adquirirem capacidade de locomoção, são muito rápidas e criativas, demorando apenas alguns segundos para ingerir produtos como materiais de limpeza, medicamentos ou plantas tóxicas.
A predisposição aos acidentes na criança somente pode ser neutralizada pelos adultos responsáveis pela sua segurança e educação. Neste contexto de valorização da prevenção das intoxicações infantis, o enfermeiro deve ser entendido como um educador apto a realizar programas educacionais em ambulatórios, creches, escolas, centros de saúde e hospitais, envolvendo pais e crianças, com o intuito de se conscientizar sobre a necessidade de prevenção dos acidentes ${ }^{(4)}$. Além do desenvolvimento de estratégias preventivas, os pais e responsáveis de recém-nascidos, lactentes e crianças devem sempre ter conhecimento sobre a localização dos centros de referência para o atendimento de vítimas de intoxicação.

As intoxicações acidentais desempenham um papel importante no contexto dos acidentes na infância, principalmente pela elevada incidência, possibilidade de seqüelas irreversíveis, custos para tratamento imediato e tardio e pelo sofrimento que provocam nas crianças e suas famílias. Independente de como - acidente acontece e de quem está mais envolvido com o fato, os sentimentos de culpa, medo, desespero são os mais percebidos e verbalizados pela família ${ }^{(6-7)}$.

Neste contexto, este estudo teve como objetivo descrever o perfil das intoxicações exógenas agudas, em crianças com faixa etária entre 0 e 12 anos de idade, atendidas no período de julho a dezembro de 2006, em um hospital da rede pública de Goiânia-GO, referência em serviços especializados nas áreas da saúde da mulher e da criança.

O desenvolvimento deste estudo torna-se oportuno devido a escassez de pesquisas que caracterizem o perfil das intoxicações exógenas agudas ocorridas no estado de Goiás, na faixa etária pediátrica. Entende-se que o conhecimento das principais características sobre esse tipo de agravo à saúde das crianças possa orientar e incrementar campanhas de prevenção de acidentes tóxicos na infância promovida pelos órgãos públicos de saúde. "Estudos detalhados podem contribuir para a adoção de ações que revertam o quadro de alta incidência observado, por meio de medidas específicas a cada fase do desenvolvimento infantil" (8). 
Siqueira KM, Brandão JR, Lima HF, Garcia ACA, Gratone FM, Brasileiro MSE. Perfil das intoxicações exógenas infantis atendidas em um hospital especializado da rede pública de Goiânia-GO. Rev. Eletr. Enf. [Internet]. 2008;10(3):662-72. Available from: http://www.fen.ufg.br/revista/v10/n3/v10n3a12.htm

Além disso, acredita-se que os profissionais de saúde e, em especial, os enfermeiros que atuam em pediatria, necessitam incorporar novos conhecimentos a respeito das intoxicações ocorridas na infância para que possam desenvolver novas estratégias de prevenção e cuidado junto às vítimas desse tipo de acidente.

\section{METODOLOGI A}

Trata-se de estudo do tipo descritivo, exploratório, com abordagem quantitativa. A coleta de dados do estudo foi desenvolvida entre dezembro de 2006 e março de 2007, em um hospital especializado em atendimentos maternos e infantis da rede pública do município de Goiânia-GO. Trata-se de uma unidade que integra a rede estadual de saúde de Goiás. Está localizado próximo ao centro da cidade de Goiânia, o que o torna acessível ao público alvo, composto por crianças e adolescentes de 0 a 19 anos e mulheres.

A instituição oferece um serviço terciário de referência em pediatria, ginecologia e obstetrícia, que conta com: 22 leitos (sendo 3 extras) em pediatria; 9 leitos em ginecologia; 25 em obstetrícia; 10 em berçário de médio risco; 11 leitos em berçário de alto risco; 2 em berçário-isolamento; 9 em Unidade de Terapia Intensiva - UTI neonatal; 10 em UTI adulto; 2 leitos para isolamento em UTI adulto; e 14 leitos destinados ao serviço de emergência pediátrica e terapia de reidratação oral.

Para coleta de dados, foi utilizado um roteiro estruturado com questões fechadas. As informações foram obtidas nas "Fichas de notificação e atendimento / Sistema Nacional de Informações Tóxico-Farmacológicas - SINITOX" arquivadas no Núcleo de Vigilância Epidemiológica do hospital selecionado para o estudo, referentes às intoxicações infantis atendidas no período de julho a dezembro de 2006.

A princípio foram selecionadas 152 fichas de notificação, mas durante avaliação criteriosa dos dados preenchidos, observou-se que, apesar de na maioria dos casos o profissional definir o acidente como "intoxicação", 31 fichas notificadas não se tratavam de casos de intoxicação e sim de exposição ou reação adversa a medicamentos. A exclusão destas fichas foi feita com base nos conceitos indicados no "Manual de Preenchimento da Ficha de Notificação e Atendimento / SINITOX", referentes aos termos intoxicação, exposição e reação adversa ${ }^{(9)}$.

Após as devidas exclusões, foram selecionadas para o estudo 121 fichas de crianças com idade entre 0 e 12 anos incompletos. Essa faixa etária foi estabelecida, pois de acordo com o Estatuto da Criança e do Adolescente - ECA, considera-se criança, para os efeitos desta Lei, a pessoa até doze anos de idade incompletos, e adolescente aquela entre doze e dezoito anos de idade.

O estudo foi iniciado após autorização do Núcleo de Vigilância Epidemiológica do Hospital Materno Infantil de Goiânia e aprovação do projeto pelo Comitê de Ética em Pesquisa da mesma instituição, protocolo de aprovação CEPHMI no 029/06.

Os dados foram analisados por estatística descritiva, sendo as freqüências dispostas em tabelas e gráficos. Para apresentação foram agrupados nas unidades: “Perfil sóciodemográfico das crianças vítimas de intoxicações" e "Caracterização dos eventos de intoxicações infantis".

\section{RESULTADOS E DI SCUSSÃO}

Os acidentes domésticos entre crianças ocorrem com freqüência e contribuem para elevar a morbimortalidade infantil. Nesse contexto, as intoxicações alcançam uma dimensão preocupante e representam um dos principais tipos de acidente envolvendo crianças $^{(8,10)}$.

Neste estudo, foram analisadas informações relativas a 121 fichas de notificação e atendimento padronizadas pelo SINITOX. A média mensal de atendimentos a casos de intoxicação infantil na instituição selecionada para o estudo foi de 20,2 atendimentos. A distribuição total de atendimentos no período de julho a dezembro de 2006, segundo o mês, é apresentada na Figura 1. 


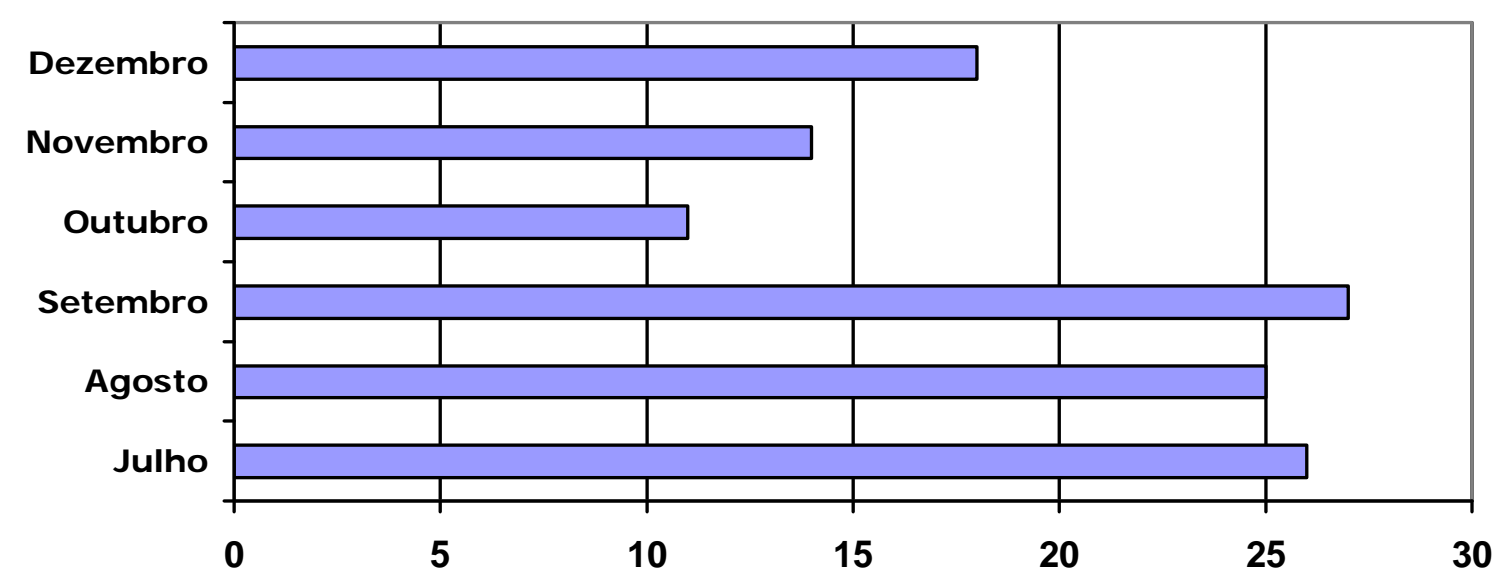

Figura 1: Distribuição de atendimentos a crianças vítimas de intoxicações endógenas, com idade entre 0 e 12 anos, em um hospital especializado da rede pública, segundo o mês. Goiânia, 2006.

No período selecionado para o estudo, observou-se que no mês de setembro ocorreu o maior número de atendimentos, seguido dos meses de julho, agosto e dezembro.

A seguir, será apresentado o perfil sóciodemográfico das crianças vítimas de intoxicação exógena caracterizado por: procedência, sexo, idade, via de exposição e agente tóxico.

\section{Perfil sócio-demográfico das crianças vítimas de intoxicações}

Quanto à procedência das crianças vítimas de intoxicações, as quais as notificações foram incluídas neste estudo, identificou-se que dos 121 casos, 110 ocorreram com crianças residentes em zona urbana, totalizando $90,9 \%$ das intoxicações, corroborando com dados similares em outros estudos ${ }^{(11-12)}$. Quanto ao município de origem, $65(53,7 \%)$ referiram ser procedentes da cidade de Goiânia-GO, capital do estado, onde se localiza a instituição selecionada para o estudo. Entretanto, um número importante de crianças era procedente de Aparecida de Goiânia-GO, 25 (20,7\%), o que se deve, provavelmente, ao fato de inexistir neste município um serviço especializado em emergências pediátricas, além da proximidade com a capital.

No que diz respeito ao gênero, observouse que a maioria dos casos de intoxicações, 62 $(51,2 \%)$, envolveu crianças do sexo masculino. Outros estudos corroboram com esse dado, ao identificarem também que intoxicações do sexo masculino são mais freqüentes ${ }^{(12-14)}$, sendo que em estudo realizado junto a 890 crianças, os casos em indivíduos do sexo masculino chegaram a totalizar $56,1 \%{ }^{(4)}$.

Esse fato pode ser compreendido sob o aspecto cultural, pois a sociedade tende a permitir que as famílias eduquem com menor vigilância as crianças do sexo masculino, sendo que os meninos adquirem liberdade de realizar atividades com menor supervisão direta de adultos mais precocemente que as meninas. Essa permissividade relacionada à educação masculina tem sido constatada no maior número de acidentes e óbitos por causas externas registrados nesse sexo ${ }^{(4,15)}$. A partir do final do primeiro ano de vida, os meninos têm o dobro de chance de sofrer injúrias do que as meninas, diferença que aumenta com a idade $^{(10)}$.

Em estudo realizado em municípios da região sul do Brasil, foi feita uma diferenciação da freqüência segundo o sexo, de acordo com o tipo de substância envolvida no episódio, os acidentes por envenenamento devido a exposição a substância nociva forma observados com maior freqüência no sexo feminino $(52,1 \%)$, enquanto que acidentes por contato com planta ou animal venenosos predominaram no sexo masculino $(68,3 \%)^{(8)}$.

Quanto à faixa etária das crianças acometidas por intoxicações exógenas, houve predomínio significativo da idade entre 1 e 3 anos, $78(64,4 \%)$, conforme a Tabela 1 . Outros estudos também trazem dados similares ${ }^{(8,12,15-}$ ${ }^{16)}$, demonstrando que as intoxicações exógenas 
Siqueira KM, Brandão JR, Lima HF, Garcia ACA, Gratone FM, Brasileiro MSE. Perfil das intoxicações exógenas infantis atendidas em um hospital especializado da rede pública de Goiânia-GO. Rev. Eletr. Enf. [Internet]. 2008;10(3):662-72. Available from: http://www.fen.ufg.br/revista/v10/n3/v10n3a12.htm

estão incluídas entre os acidentes mais freqüentes em crianças menores de 5 anos. Em 2004 foram registrados 81828 casos de intoxicação humana no Brasil, sendo a maioria dos casos por medicamentos (23700) e as maiores letalidades nos casos de intoxicação por agrotóxicos de uso agrícola e raticidas (155), sendo que a faixa etária mais acometida foi a de 1 a 4 anos, totalizando $21,88 \%$ dos casos em humanos $^{(3)}$.

Tabela 1: Crianças vítimas de intoxicações exógenas atendidas no Hospital Materno Infantil de Goiânia, segundo idade. Goiânia, 2006.

\begin{tabular}{cccc}
\hline Idade & $\mathrm{N}$ & $\%$ & 6,6 \\
\hline$<$ que 1 & 8 & 64,4 & 20,7 \\
1 a 3 & 78 & 8,3 & 0 \\
4 a 6 & 25 & 100 \\
7 a 9 & 10 & 0 & \\
10 a $12 *$ & 121 &
\end{tabular}

Pelas características próprias do seu desenvolvimento, as crianças nessa faixa etária se tornam mais hábeis e tendem a explorar o ambiente, podendo encontrar materiais de limpeza, medicamentos e outros produtos de uso doméstico, os quais se encontram inadequadamente armazenados. Além disso, começam a subir em móveis, abrindo armários e alcançando prateleiras e gavetas ${ }^{(7-8,15)}$.

Segundo dados do SINITOX ${ }^{(3)}$, crianças menores de 5 anos representam aproximadamente $35 \%$ dos casos de intoxicação por medicamentos no Brasil. O que pode estar relacionado à curiosidade quanto ao conteúdo de potes, vidros fechados com líquidos coloridos e pastilhas ${ }^{(7)}$. Nesse sentido, alguns fatores podem ser entendidos como contribuintes para a elevada ocorrência de acidentes por intoxicação medicamentosas entre crianças, como o gosto agradável de alguns medicamentos, o formato atraente, a facilidade de violação das embalagens, além da prática da automedicação, acompanhada pelo armazenamento inadequado de medicamentos $^{(8)}$.

Deste modo, no sentido de prevenir as intoxicações com medicamentos e produtos de limpeza, são feitas recomendações de que as embalagens ofereçam resistência que dificultem a abertura de frascos por crianças, além de serem sempre guardadas em locais de difícil acesso $^{(3,14)}$. O Projeto de lei no. 4841-A/94, em tramitação no Congresso Nacional, visa a adoção do EEPC (Embalagem Especial de Proteção à Criança, implementada nos Estados
Unidos e Canadá) em nosso país, que deverá ser confeccionada de modo que seja significativamente difícil para criança com menos de 5 anos de idade, abri-la ou retirar uma quantidade tóxica ou perigosa do produto nela contida ${ }^{(3)}$.

Estudo realizado na Paraíba, junto a mães de crianças menores de 5 anos, identificou que a maioria das mães costumam guardar medicamentos e materiais de limpeza em locais ao alcance da criança, tais como embaixo da cama ou na área de serviço em armários próximos ao chão e que não possuem uma fechadura de segurança(7).

Assim, faz-se necessário que os profissionais de saúde intensifiquem ações de educação em saúde voltadas para a prevenção de acidentes na infância, especialmente, as intoxicações infantis ocorridas em ambiente doméstico. É importante envolver a família da criança nestas ações preventivas, enfocando que estes acidentes são sempre evitáveis e que não devem ser considerados como situações inerentes à infância.

\section{Caracterização dos eventos de intoxicações infantis}

No que diz respeito ao local de ocorrência, a maioria das crianças vítimas de intoxicações estavam em suas residências no momento do acidente $(80,2 \%)$, dado similar ao encontrado na literatura ${ }^{(11,14,17)}$. No presente estudo não foi possível identificar quem era o adulto responsável pela criança no momento da intoxicação, pois essa informação não consta 
Siqueira KM, Brandão JR, Lima HF, Garcia ACA, Gratone FM, Brasileiro MSE. Perfil das intoxicações exógenas infantis atendidas em um hospital especializado da rede pública de Goiânia-GO. Rev. Eletr. Enf. [Internet]. 2008;10(3):662-72. Available from: http://www.fen.ufg.br/revista/v10/n3/v10n3a12.htm

nas fichas de notificação, fontes da coleta de dados. Porém, estudo realizado no município de Porto Alegre-RS, Brasil, comprovou que os pais eram os responsáveis pelas crianças vítimas de intoxicações em $76,2 \%$ dos eventos ${ }^{(14)}$.

Sabe-se que a presença do adulto não impede que o acidente ocorra, o que pode estar relacionado ao desconhecimento dos riscos e aspectos preventivos, ou ainda por não realizar uma supervisão direta da criança, isto é, encontra-se presente, porém realizando outras atividades $^{(4)}$. Estudo australiano sobre 576 casos de intoxicações infantis revelou que, na maioria dos eventos, as crianças estavam sem supervisão direta dos responsáveis $(61,6 \%)$, os quais estavam próximos, mas em $50,6 \%$ dos casos, ocupados com outras atividades domésticas $^{(17)}$.

Uma observação importante é de que "pais se preocupam em excesso com a iminência de perigos difíceis de ocorrer, tais como seqüestros, roubo, desaparecimento; desconsiderando que os perigos mais freqüentes estão presentes nas situações de vivência cotidiana da criança" ${ }^{\prime(7)}$.

Um dos itens avaliados na caracterização dos eventos de intoxicações foi a via de exposição, que segundo a "Ficha de notificação e atendimento / Sistema Nacional de Informações Tóxico-Farmacológicas - SINITOX ", pode ser classificada em via oral, cutânea, respiratória, parenteral, nasal, ocular, retal, vaginal, transplacentária, aleitamento materno ou mordedura/picada. No presente estudo a via oral foi a mais freqüente, sendo exclusiva em 116 casos $(95,9 \%)$ e, em dois casos, com concomitante intoxicação por via respiratória. Houve apenas um caso de intoxicação por via parenteral e em dois registros essa informação foi ignorada.

A via oral é a via de exposição que mais caracteriza os eventos de intoxicações infantis ${ }^{(14,16)}$. Deste modo, torna-se importante ressaltar os aspectos preventivos destes acidentes como acondicionamento adequado de produtos de limpeza, medicamentos e agentes tóxicos; evitar a reutilização de embalagens que possam confundir o adulto e chamar a atenção da criança; evitar comparações entre medicamentos e alimentos agradáveis como balas e doces; utilizar embalagens que impeçam a abertura por crianças; e, sobretudo, supervisionar diretamente a criança durante suas atividades de lazer.

Outro item avaliado neste estudo foi o tipo de exposição, que pode ser classificado em: aguda única, aguda repetida, crônica e aguda sobre crônica. Todas as intoxicações foram classificadas como aguda única, excetuando-se 16 casos $(13,2 \%)$, onde o profissional de saúde responsável pelo preenchimento da ficha de notificação do evento ignorou esse item. No entanto, avaliar essa informação pode ser importante na identificação de repetições de injúrias contra a criança. Há evidências de que a repetição de eventos traumáticos está associada com pelo menos um fator de risco socioambiental, como abuso de drogas, mãe adolescente, cuidador solteiro, cuidador com doença mental e história de violência intrafamiliar ${ }^{(18)}$.

Neste contexto, o enfermeiro pode assumir importante papel como educador, especialmente, durante as consultas de enfermagem voltadas para saúde da criança, informando pais e responsáveis sobre os principais riscos aos quais as crianças podem ser expostas, assim como, formas de prevenir acidentes e condutas a serem tomadas diante da ocorrência dos mesmos.

No presente estudo, no que diz respeito ao agente tóxico, os medicamentos se destacaram quanto à freqüência, totalizando 41 casos $(34 \%)$, seguido das intoxicações por raticidas, 17 ( $14 \%)$, e produtos químicos de uso domiciliar, 16 (13\%), conforme demonstrado na Figura 2. Dentre estes últimos, destaca-se os acidentes envolvendo ingestão de água sanitária pelas crianças, os quais totalizaram seis casos. Esse produto químico tem como principal componente o hipoclorito de sódio, encontrado como o agente causal de maior incidência $(46 \%)$ em estudo sobre 50 casos de acidentes infantis por produtos domissanitários em Maringá-PR, Brasil ${ }^{(10)}$. 


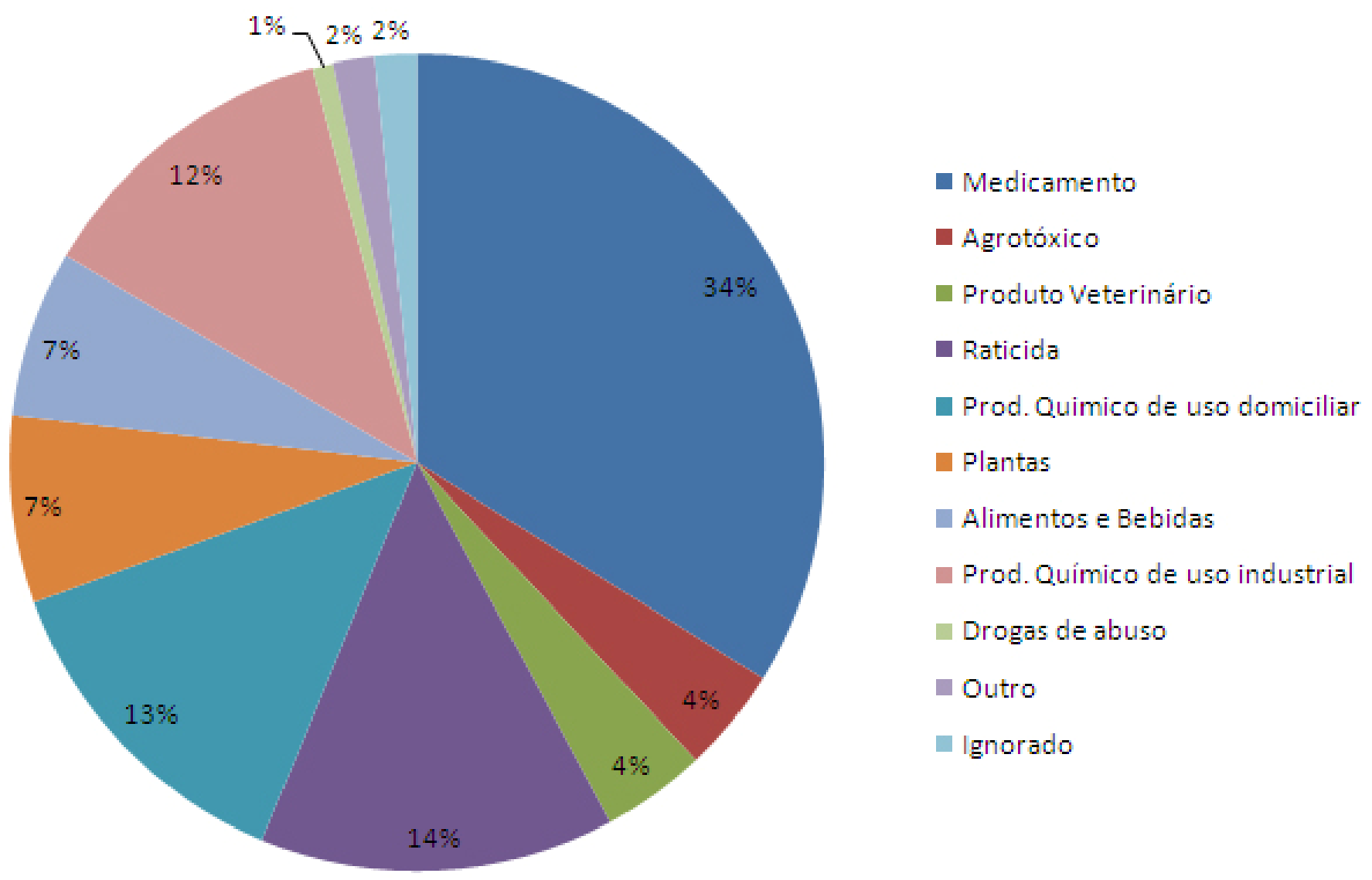

Figura 2: Distribuição dos casos de intoxicação infantil entre crianças de 0 a 12 anos, atendidos no Hospital Materno Infantil, segundo o agente tóxico. Goiânia, 2006.

No Brasil, como na maioria dos países, os medicamentos se apresentam com o principal agente tóxico, respondendo por, aproximadamente, $28 \%$ dos casos de intoxicação humana registrados anualmente pelo SINITOX, sendo que crianças menores de 5 anos representam, aproximadamente, 35\% destes casos. Os benzodiazepínicos, antigripais, anti-depressivos e antiinflamatórios são as classes responsáveis pela maioria das intoxicações por medicamentos em nosso país ${ }^{(3)}$.

O padrão de consumo de medicamentos da população brasileira pode contribuir para que as intoxicações por este tipo de agente aconteçam com maior freqüência. Esse padrão é caracterizado por grande utilização de fármacos sintomáticos e automedicação. Acredita-se que armazenar inadequadamente grandes quantidades de medicamentos pertencentes às mais variadas classes farmacológicas e o hábito medicar-se excessivamente no ambiente domiciliar favoreçam esses acidentes junto à população infantil ${ }^{(15)}$.
No presente estudo, os raticidas representaram o segundo grupo de agente tóxico mais freqüente nas intoxicações exógenas agudas infantis, enquanto que em estudo brasileiro envolvendo 10.645 indivíduos entre 0 e 14 anos vítimas de intoxicações, os raticidas ocuparam o terceiro lugar entre os grupos com maiores registros ${ }^{(12)}$. Esse dado é preocupante devido a alta toxicidade deste agente que provoca alterações como aumento da fragilidade capilar e manifestações clínicas como náuseas, vômitos, gengivorragias, epistaxe, hematêmese, hematomas, equimoses, hematúria e melena. Em casos de intoxicação severa ocorre hemorragia maciça, dor abdominal intensa e aguda, choque e coma, podendo levar à morte ${ }^{(12)}$.

Outro dado que chama a atenção no que diz respeito ao agente causal das intoxicações infantis em estudo é o número de acidentes envolvendo produtos químicos de uso industrial, dentre os quais destaca-se a ingestão de soda cáustica por sete crianças. As conseqüências relacionadas à morbidade deste tipo de acidente 
Siqueira KM, Brandão JR, Lima HF, Garcia ACA, Gratone FM, Brasileiro MSE. Perfil das intoxicações exógenas infantis atendidas em um hospital especializado da rede pública de Goiânia-GO. Rev. Eletr. Enf. [Internet]. 2008;10(3):662-72. Available from: http://www.fen.ufg.br/revista/v10/n3/v10n3a12.htm

devem ser consideradas, pois além de necessitar de terapêutica prolongada, com custos elevadas para o serviço público de saúde, o sofrimento causado à criança e familiares tornam o tratamento e o processo de reabilitação ainda mais difíceis.

No geral, as manifestações clínicas mais comumente apresentadas pelas 121 crianças vítimas de intoxicações foram vômitos, presentes em 48 casos (39,7\%); sonolência, 25 $(20,7 \%)$; sialorréia, 22 (18,2\%); palidez, 13 $(10,8 \%)$; e agitação, 13 (10,8\%). Ressalta-se que algumas crianças apresentaram mais desses sinais e sintomas relacionados ao quadro de intoxicação. Quanto ao tratamento realizado, à maioria das crianças vítimas de intoxicação foram propostos: observação clínica e tratamento sintomático. Também foram realizados com relativa freqüência: lavagem gástrica, diluição e tratamento com carvão ativado.

A manifestação clínica de vômito foi a mais apresentada nos casos de intoxicação analisados neste estudo. O vômito é um processo reflexo altamente coordenado que pode ser precedido por um aumento da salivação e começa com esforços involuntários para vomitar. Este processo é coordenado no centro bulbar do vômito, o qual é influenciado diretamente pela inervação aferente e indiretamente pela zona do gatilho quimiorreceptora e centros superiores do sistema nervoso central. Muitos processos agudos e crônicos podem causar vômitos, sendo que as intoxicações seriam uma das etiologias agudas mais comuns em crianças ${ }^{(19)}$.

Dentre os agentes causais das intoxicações que provocam, mais comumente, a manifestação clínica de sonolência, pode-se citar os antidepressivos cíclicos e os antidepressivos inibidores da recaptação da serotonina. A classe dos cíclicos bloqueia a recaptação neuronal de norepinefrina, 5hidroxitriptamina, serotonina e dopamina nos sistemas nervosos central e periférico, também produzem graus variáveis de sedação e efeitos alfa-bloqueadores e anticolinérgicos. $O$ padrão de efeitos tóxicos é diferente entre adultos e crianças, sendo a sonolência e as convulsões os mais comuns em pediatria, podendo inclusive evoluir para letargia ou coma em até um terço dos casos pediátricos ${ }^{(19)}$.

Nos casos de intoxicação por antidepressivos inibidores da recaptação da serotonina (como por exemplo, a fluoxetina) em crianças, os efeitos mais comumente encontrados são sonolência ou hiperatividade e agitação. Podendo também ocorrer tremores, náuseas, vômitos e dor abdominal ${ }^{(19)}$.

Cabe ressaltar que, considerando a necessidade de estruturar e organizar a prestação da informação toxicológica e da assistência a indivíduos intoxicados, bem como qualificar os dados epidemiológicos relacionados a esses agravos; a necessidade de definir critérios para o reconhecimento dos centros já existentes e de estabelecer parâmetros para a criação de novos centros no Brasil, foi criada pela Agência Nacional de Vigilância Sanitária ANVISA, a Rede Nacional de Centros de Informação e Assistência Toxicológica RENACIAT e aprovadas as "Diretrizes para qualificação dos centros de informação e assistência toxicológica"(20).

Observou-se, durante a coleta de dados, que muitas condutas terapêuticas foram sustentadas por informações ou avaliações dos profissionais do Centro de Informações Toxicológicas (CIT) de Goiás, serviço que tem como função fornecer informação e orientação sobre o diagnóstico, prognóstico, tratamento e prevenção das intoxicações e envenenamentos, assim como sobre a toxicidade das substâncias químicas e biológicas e os riscos que elas ocasionam à saúde $\mathrm{e}^{(20)}$.

\section{CONCLUSÃO}

O objetivo deste estudo foi descrever o perfil das intoxicações exógenas agudas, em crianças com faixa etária entre 0 e 12 anos de idade, atendidas em um hospital especializado em atendimentos maternos e infantis da rede pública de Goiânia no período de julho a dezembro de 2006.

A análise dos dados demonstrou que a maioria das crianças é procedente de zona urbana, sendo 53,7\% de Goiânia-GO, município onde se localiza a instituição do estudo. Dentre as 121 intoxicações infantis, 51,2\% corresponderam a acidentes com indivíduos do 
Siqueira KM, Brandão JR, Lima HF, Garcia ACA, Gratone FM, Brasileiro MSE. Perfil das intoxicações exógenas infantis atendidas em um hospital especializado da rede pública de Goiânia-GO. Rev. Eletr. Enf. [Internet]. 2008;10(3):662-72. Available from: http://www.fen.ufg.br/revista/v10/n3/v10n3a12.htm

sexo masculino, sendo que em $80,2 \%$ dos casos a criança estava em seu domićlio no momento do acidente.

A faixa etária onde as intoxicações foram mais freqüentes correspondeu a crianças entre 1 e 3 anos, totalizando $64,4 \%$ dos casos notificados. Essas crianças possuem características próprias que as tornam mais propensas a esse tipo de acidente, além disso, as peculiaridades do ambiente doméstico e a vigilância dos adultos guardam relação direta com a freqüência das intoxicações nesta faixa etária.

No presente estudo a via oral foi a mais freqüente, sendo exclusiva em 116 casos $(95,9 \%)$ e, em dois casos, com concomitante intoxicação por via respiratória. O agente causal mais prevalente foi o medicamento (34\%), seguido dos raticidas $(14 \%)$ e produtos químicos de uso domiciliar (13\%).

Acredita-se que a redução dos índices de incidência das intoxicações infantis pode ser alcançada por ações educativas baseadas em estudos regionalizados, realizados durante períodos de tempo mais prolongados, que caracterizem melhor o perfil destas intoxicações e subsidiem a implantação de programas de prevenção específicos.

Faz-se necessária a mobilização de diferentes segmentos da população com a finalidade de assegurar às crianças e suas famílias informações que envolvam aspectos preventivos e terapêuticos das intoxicações, reduzindo índices de mortalidade e minimizando aspectos relativos à morbidade deste tipo de acidente infantil.

Neste contexto, o enfermeiro assume um papel fundamental, atuando como educador junto aos pais e crianças; em momentos diversos como educação de gestantes e puérperas; no atendimento ambulatorial de crianças, como consultas de enfermagem em puericultura; na assistência em creches e escolas; durante o período de hospitalização das crianças; enfim, usando esses momentos para a implementação de programas de prevenção de acidentes infantis em geral e, principalmente, de intoxicações exógenas agudas.

\section{REFERÊNCI AS}

1. TabNet Win32 2.7: Óbitos por Causas Externas - Brasil [Internet]. Brasília: Ministério da Saúde (BR) [update 2007 mai 01, cited 2007 mai 04]. Informações de saúde, estatísticas vitais, mortalidade e nascidos vivos. Available from:

http://tabnet. datasus.gov. br/cgi/tabcgi.exe?sim /cnv/extuf. def.

2. Gawryszewski VP, Koizumi MS, Mello-Jorge MHP. As causas externas no Brasil no ano 2000: comparando a mortalidade e a morbidade. Cadernos de Saúde Pública. 2004;20(4): 9951003.

3. Sistema Nacional de Informações TóxicoFarmacológicas - SINITOX [Internet]. Brasília: Ministério da Saúde - Fundação Oswaldo Cruz (BR) [update 2007 mai 01, cited 2007 mai 04]. Casos registrados de intoxicação humana e envenenamento. Available from:

http://www. fiocruz.br/sinitox/

4. Filócomo FRF, Harada MJCS, Silva CV, Pedreira MLG. Estudo dos acidentes na infância em um pronto socorro pediátrico. Revista Latino-Americana de Enfermagem. 2002; 10(1):41-7.

5. Schvartsman C, Schvartsman S. Intoxicações exógenas agudas. Jornal de Pediatria. 1999; 75(2): 244-50.

6. Ozdogan H, Davutoglu M, Bosnak M, Tutanc $M$, Haspolat K. Pediatric poisonings in southeast of Turkey: epidemiological and clinical aspects. Hum Exp Toxicol. 2008; 27(1): 45-8.

7. Reichert APS, Silva SLF, Costa TNA. Prática de mães na prevenção de acidentes na infância com crianças de 0 a 5 anos de idade. Revista Nursing. 2003;62(6):33-7.

8. Martins CBG, Andrade SM, Paiva PAB. Envenenamentos acidentais entre menores de 15 anos em município da Região Sul do Brasil. Cadernos Saúde Pública. 2006;22(2):407-14.

9. Ministério da Saúde; Fundação Oswaldo Cruz, Sistema Nacional de Informações TóxicoFarmacológicas - SINITOX. Manual de preenchimento da ficha de notificação e de atendimento. Rio de Janeiro (Brasil): FIOCRUZ; 2003.

10. Blank D. Controle de injúrias sob a ótica da pediatria contextual. Jornal de Pediatria. 2005; 81( 5 Suppl):S123-36. 
11. Ferreira A, Borelli E, Casoni A, Santos FM, Oliveira MLF. Acidentes infantis domésticos por produtos domissanitários registrados em centro de assistência toxicológica da Região Sul. Espaço para a Saúde [Internet]. 2001 [cited 2006 out 24];3(1):56-69. Available from: http://www.ccs.uel.br/espacoparasaude/v3n1/i ndicel.htm.

12. Segura-Muñoz SI, Julião FC, Takayanagui AMM, Galvis-Alonso OY, Mello DF. Condições de saúde ambiental e infância: análise das intoxicações por agentes químicos no Brasil. O Mundo da Saúde. 2005;29(2): 199-206.

13. Paes CEN, Gaspar VLV. As injúrias não intencionais no ambiente domiciliar: a casa segura. Jornal de Pediatria. 2005;81(5 Suppl):S146-54.

14. Ramos CLJ, Targa MBM, Stein AT. Perfil das intoxicações na infância atendidas pelo Centro de Informação Toxicológica do Rio Grande do Sul (CIT/RS), Brasil. Cadernos Saúde Pública. 2005;21(4): 1134-41.

15. Alcântara DA, Vieira LJES, Albuquerque VLM. Intoxicação medicamentosa em criança. Revista Brasileira em Promoção da Saúde. 2003; 16(1/2): 10-16.

16. Bucaretchi F, Dragosavac S, Vieira RJ. Exposição aguda a derivados imidazolínicos em crianças. Jornal de Pediatria. 2003;79(6):51924.

17. Ozanne-Smith J, Day L, Parsons B, Tibbals J, Dobbin M. Childhood poisoning: access and prevention. Journal of Paediatrics and Child Health. 2001; 37(3): 262-5.

18. Braun PA, Beaty BL, Diguiseppi C, Steiner $J F$. Recurrent early childhood injuries mong disadvantaged children in primary care settings. Injury Prevention. 2005; 11:251-5.

19. Behrman RE, Kliegman RM, Jenson HB. Nelson - Tratado de Pediatria. 16a edição. Rio de Janeiro: Guanabara Koogan; 2002.

20. Ministério da Saúde; Agência Nacional de Vigilância Sanitária. Resolução da Diretoria Colegiada - RDC №. 19. Brasília (Brasil): Ministério da Saúde; 2005. 